\title{
A inserção do nutricionista na Atenção Básica: uma proposta para o matriciamento da atenção nutricional
}

\author{
The inclusion of nutritionists in Primary Health Care: \\ a proposal for a matrix model of nutritional care
}

Marina Borelli ${ }^{1}$

Semíramis Martins Álvares Domene ${ }^{2}$

Laís Amaral Mais ${ }^{1}$

Juliana Pavan ${ }^{1}$

José Augusto de Aguiar Carrazedo Taddei ${ }^{1}$
Departamento de Pediatria, Universidade Federal de São Paulo (UNIFESP). R. Loefgreen 1647, Vila Clementino. 04040-032. São Paulo SP Brasil. marinaborellip@gmail.com ${ }^{2}$ Departamento de Políticas Públicas e Saúde Coletiva, UNIFESP.

\begin{abstract}
Nutritionists were integrated into the Family Health Support Centers back in 2008. Focusing on the development of technical and pedagogical support and assistance to Family Health Teams, the aim of this study was to develop a proposal for a matrix model for nutrition aimed at women and children in the Family Health Strategy. The study was conducted in a Basic Health Unit and was divided into Cycle I, including interviews with families of 0 to 6-year-old children to establish the confines of the territory; and Cycles II and III, with the development of intersectorial field activities to promote food and nutritional security based on matrix support. Following the diagnosis of social vulnerability, children's nutritional risk, indicators of early weaning and inadequate food intake, actions were developed in order to propose a matrix model of nutritional measures in three scenarios, namely at the community, family and individual level. Among the activities that were developed, the main ones included cooking classes, prenatal care, and the training of Community Health Agents. This study tested ways to develop nutritional care by broadened clinical action and territory recognition, seeking to enhance the activities of nutritionists as health educators.
\end{abstract}

Key words Nutritionist, Primary Health Care, Family Health Program
Resumo Em 2008 houve a integração do nutricionista nos Núcleos de Apoio à Saúde da Família. Com foco no desenvolvimento de apoio assistencial e técnico-pedagógico às Equipes de Saúde da Família, o objetivo do trabalho foi desenvolver uma proposta de ações matriciais de nutrição dirigidas à mulher e à criança na Estratégia de Saúde da Família. O trabalho foi desenvolvido em uma Unidade Básica de Saúde e dividido em Ciclo I, a partir das entrevistas com famílias de crianças de 0 a 6 anos para compor o reconhecimento do território; e Ciclos II e III, a partir do desenvolvimento de atividades intersetoriais de campo para promover a segurança alimentar $e$ nutricional com base no apoio matricial. Com o diagnóstico de vulnerabilidade social, risco nutricional das crianças, indicadores de desmame precoce e consumo alimentar inadequado, foram desenvolvidas ações com o objetivo de propor um modelo matricial de ações de nutrição em três cenários: comunidade, família e indivíduo. Dentre as atividades desenvolvidas destacam-se oficinas culinárias, atenção ao pré-natal e capacitação de agentes comunitários de saúde. Este trabalho testou formas de desenvolver assistência nutricional a partir da clinica ampliada e reconhecimento do território, visando aprimorar a atuação do nutricionista como educador em saúde.

Palavras-chave Nutricionista, Atenção primária à saúde, Programa saúde da família 


\section{Introdução}

O Programa de Saúde da Família - PSF surge no Brasil em 1994 como uma estratégia de reorientação do modelo assistencial em saúde a partir da Atenção Básica - AB, mediante a implantação de equipes multiprofissionais em Unidades Básicas de Saúde - UBS ${ }^{1,2}$. O principal propósito do PSF, atualmente denominado Estratégia de Saúde da Família - ESF, é reorganizar a prática de atenção à saúde em novas bases e substituir o modelo tradicional, descentralizando os serviços de acordo com as necessidades da população, o que representa democratização das instituições de saúde ao prever a participação popular, incluindo a família na abordagem do binômio saúde-doença e passando a enfocá-la em seu ambiente físico e social $^{2-5}$.

Como parte das ações para qualificação da assistência, em 2008, o Ministério da Saúde (MS) criou os Núcleos de Apoio à Saúde da Família NASF; assim, mais profissionais de saúde, entre eles o nutricionista, passaram a integrar a $\mathrm{AB}$ a partir de ações de apoio matricial à Equipe de Saúde da Família (SF). O matriciamento é uma proposta de atuação em que as competências compartilhadas em diferentes níveis de atenção promovem integração das ações de saúde, de modo a desenvolver apoio assistencial e técnico-pedagógico às Equipes de SF; a partir das demandas identificadas conjuntamente, as equipes multiprofissionais atuam de forma integrada com as Equipes de SF. Ao NASF cabe o apoio, a ampliação e o aperfeiçoamento da atenção e da gestão da saúde na $\mathrm{AB}$, de forma a qualificar a assistência e complementar competências a partir do conceito de núcleo e campo ${ }^{6-9}$.

Tendo em vista a implantação dos NASF, em 2008, experimentou-se acelerada expansão: em junho de 2011 existiam 1388 NASF implantados no país ${ }^{10}$, que contavam com 1038 nutricionistas. Este crescimento representa uma oportunidade para organizar ações de nutrição, necessárias frente à alteração dos padrões alimentares experimentada no Brasil a partir dos anos 1980, que convive com a desnutrição expressa por déficit de crescimento e carências nutricionais por micronutrientes, paralelamente ao aumento da obesidade e de outras doenças crônicas não transmissíveis (DCNT), condições agravadas pela queda na qualidade do repertório alimentar ${ }^{11}$. A presença do nutricionista na $\mathrm{AB}$ fortalece esta oportunidade à medida que qualifica as ações de atenção nutricional, previstas na Política Nacional de Alimentação e Nutrição (PNAN) ${ }^{12}$.
Com a perspectiva de atuação em rede, as ações de assistência nutricional junto à ESF têm como foco: promoção de ações integrativas e intersetoriais de educação em saúde e nutrição, realização do cuidado nutricional em todas as fases do curso da vida, desenvolvimento de planos terapêuticos para DCNT e deficiências nutricionais e estímulo à produção e ao consumo de alimentos saudáveis, ações garantidas pela PNAN ${ }^{12}$, afirmadas pelo Sistema Único de Saúde (SUS) ${ }^{13}$ e reafirmadas pela proposta da agenda única da nutrição ${ }^{14}$. Ao considerar a formação clássica dos profissionais de saúde e a modesta participação do nutricionista na $\mathrm{AB}$, desde a regulamentação da profissão no país em 1967, o objetivo deste trabalho foi desenvolver uma proposta para o matriciamento das ações de nutrição dirigidas à mulher e à criança no âmbito da ESF.

\section{Métodos}

A partir do contato com a equipe da Unidade Básica de Saúde Perseu Leite de Barros (UBS-PLB), localizada no Distrito Noroeste do município de Campinas, SP e da formalização da parceria com a Secretaria Municipal de Saúde, o trabalho teve início com o reconhecimento do território da área de abrangência da UBS, nos anos de 2009 e 2010. Os dados populacionais disponíveis no Censo ${ }^{15}$ foram empregados para sorteio dos setores censitários de acordo com seu peso e então realizada amostra aleatória simples dentro de cada setor, para identificação das residências a serem visitadas.

O protocolo foi concebido para viabilizar a obtenção de dados epidemiológicos suficientes à compreensão da dinâmica relacionada à alimentação e à nutrição em três cenários, onde a atenção nutricional tem lugar: comunidade, família e indivíduo ${ }^{9,16}$, tendo como foco a Nutrição Materno-Infantil. Esta opção metodológica considerou a experiência dos pesquisadores com a temática, prevista entre as principais preocupações da Política Nacional de Atenção Básica 5 .

O estudo foi dividido nas seguintes fases: a fase inicial das atividades de reconhecimento do território, representada pelo ciclo I do modelo proposto e a fase de desenvolvimento das atividades de campo da proposta de apoio matricial, representada pelos ciclos II e III do modelo para matriciamento da atenção nutricional.

Foram entrevistadas famílias com crianças de 0 a 6 anos, por meio da aplicação de um questionário estruturado e pré-codificado sobre condi- 
ções sociodemográficas, de gestação, aleitamento materno e introdução de alimentos e avaliação antropométrica, além do consumo alimentar mensal da família.

Em 2009 e 2010, foram entrevistadas 210 e 129 famílias, respectivamente; 11 famílias não foram incluídas por pelo menos um dos seguintes motivos: ausência de dados no questionário, criança portadora de deficiência, crianças que não residiam na área de cobertura, criança adotiva e responsável que não soube informar dados gestacionais, de aleitamento materno e alimentação complementar. Desta forma, a população de estudo foi composta por 328 crianças entre 0 e 6 anos, com uma perda amostral total de apenas $3,35 \%$.

A partir do diagnóstico da situação de saúde e de nutrição da população, do estabelecimento de vínculo com as Equipes de SF e com a rede de apoio (Centro de Referência à Assistência Social, creche, escola de ensino fundamental e igrejas), foram concebidas as atividades de campo para o desenvolvimento da proposta de matriciamento.

As opções metodológicas que subsidiaram a construção deste modelo estão apoiadas em dois eventos de grande prevalência e para os quais a atenção nutricional traz resolubilidade: desvios nutricionais (subnutrição, carências nutricionais específicas e excesso de peso) e desmame precoce. O estudo dos seus determinantes foi central para a compreensão das oportunidades de atenção nutricional no âmbito da UBS, e subsidiou o desenho dos processos de trabalho apoiados na identificação das competências do nutricionista. O modelo desenvolvido foi testado por 12 meses na UBS.

O projeto foi financiado pela Fundação de Amparo à Pesquisa do Estado de São Paulo FAPESP e aprovado por Comitê de Ética de Pesquisa com Seres Humanos da Universidade Federal de São Paulo - UNIFESP.

\section{Resultados e discussão}

\section{Resultados que subsidiaram o modelo de matriciamento}

Os dados referentes à condição socioeconômica das famílias, ao estado nutricional das crianças avaliadas, às condições de pré-natal e aleitamento materno e ao consumo alimentar familiar constituíram a etapa de reconhecimento do território que subsidiou o desenvolvimento pela equipe do projeto das atividades de matriciamento propostas no modelo e de sua aplicação.
A média de idade das crianças estudadas foi de $31,5( \pm 19,05)$ meses. Dentre as 328 crianças, $54 \%$ eram do sexo masculino; $36,6 \%$ das famílias residiam em moradias alugadas ou cedidas; 40,85\% das famílias recebiam menos do que 0,5 salário-mínimo per capita, e 19,5\% das mães eram chefes de família; $28,7 \%$ das famílias recebiam apoio de programas oficiais, sendo o Bolsa Família o principal. Estes dados indicam a vulnerabilidade da população da área de cobertura ${ }^{9,17}$.

A avaliação do estado nutricional das crianças apontou que $7 \%$ das crianças apresentaram baixa estatura (z-escore estatura/idade $<-2$ ); 2,1\% magreza (z-escore IMC/idade < -2); 9,7\% excesso de peso (z-escore IMC/idade $>2$ ); $18 \%$ das crianças apresentaram risco para sobrepeso, segundo o indicador IMC/idade $(1<\mathrm{z}$-escore $\leq$ 2), o que chama a atenção para situação de risco nutricional neste grupo (Tabela 1). Este perfil antropométrico reproduz aquele encontrado no país: o déficit de estatura está próximo da média nacional $(6,95 \%)$ e o excesso de peso é maior que a média para o país $(7,3 \%)^{17}$. A carência de protocolos para acompanhamento de peso e altura em equipes de saúde também foi identificada em estudo que avaliou as ações em nutrição na ESF em São Paulo $^{18}$. A sistematização desta rotina cresce em relevância ao se considerar a oportunidade de alimentação do Sistema Nacional de Vigilância Alimentar e Nutricional - SISVAN-web, que inclui, adicionalmente, marcadores dietéticos, além de ser atividade preconizada pela $\mathrm{PNAN}^{12}$.

O nutricionista encontra uma oportunidade para ações de promoção do aleitamento materno (AM) no período pré-natal, dada a média de comparecimento de 10 consultas; contudo, $12,7 \%$ das mães iniciaram o pré-natal após o $2^{\circ}$ trimestre de gestação, o que indica a adoção de abordagem especial para este grupo; $54,3 \%$ das crianças nasceram de parto cesárea, uma situação que pode comprometer o estabelecimento da lactação nos primeiros dias de vida. A mediana nacional de 1,8 meses para aleitamento materno exclusivo (AME) é inferior a mediana de 3 meses encontrada neste estudo; a mediana de 8 meses para aleitamento materno total (AMT) observada está muito abaixo da mediana nacional de 11,2 meses $^{19}$. Adicionalmente, a frequente oferta de alimentos no segundo e terceiro meses de vida ratificam a relevância da atenção nutricional dirigida às mães no período pré-natal, evitando-se a percepção tardia da interrupção do AME por parte da equipe de saúde, o que ocorre no atendimento de puericultura, em época de difícil reversão. 


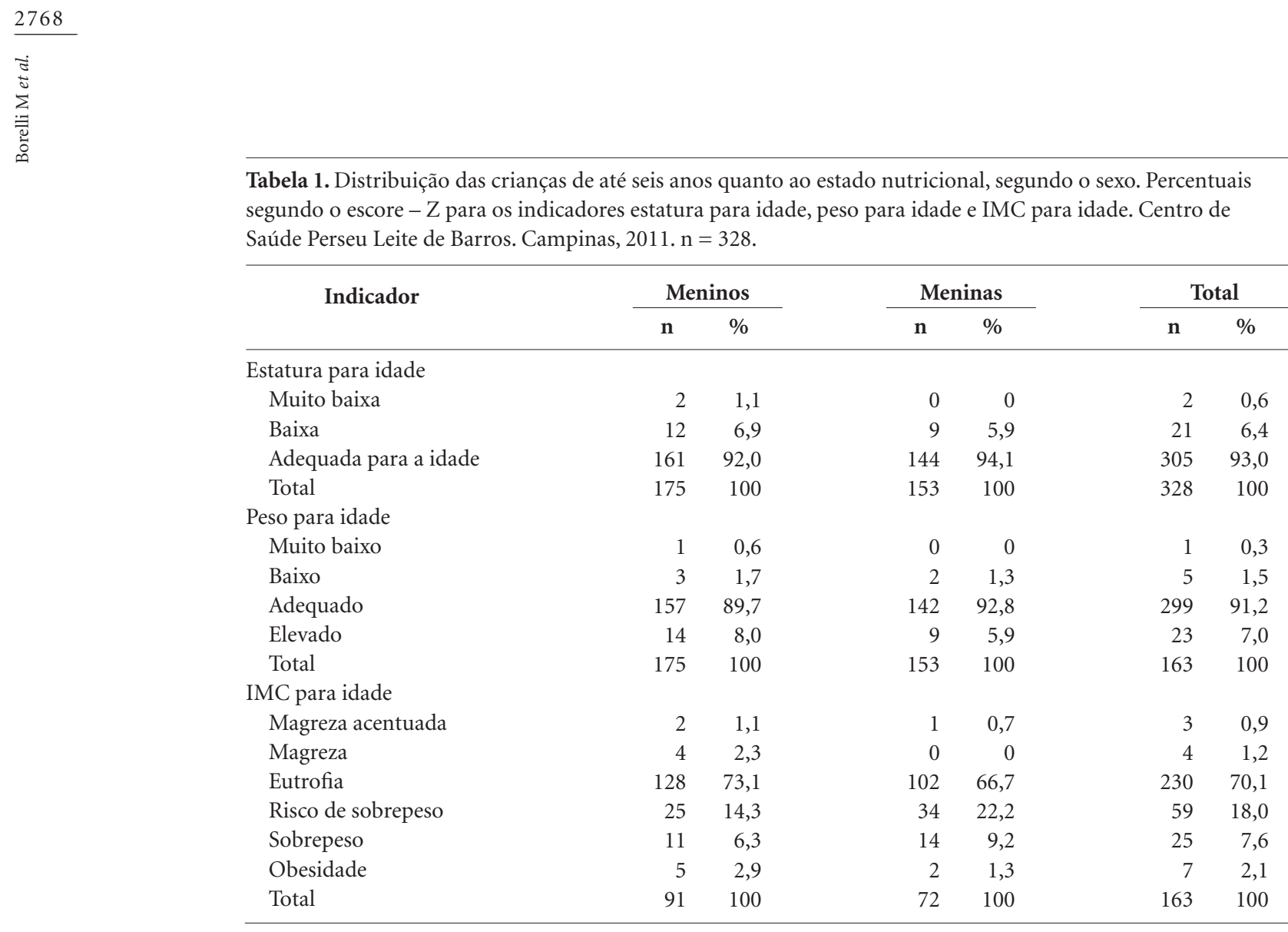

Para o estudo do consumo alimentar familiar, foram avaliados os dados referentes à aquisição alimentar (em Kg per capita/ano), organizados de forma a serem comparáveis com as Pesquisas de Orçamento Familiar - POF, em 19 grupos de alimentos ${ }^{11}$. Considerou-se o consumo segundo a renda, estimada em Salários Mínimos Familiares Per Capita - SMFPC, nos grupos SMPFC 1 (indigentes), SMPFC 2 (pobres) e SMPFC 3 (não pobres ou indigentes), adotando-se para esta classificação critério que considera um conjunto de dados para estabelecimento de uma cesta de consumo $^{20}$. Estes dados subsidiaram as atividades de oficina de alimentação saudável e oficina culinária com as famílias, além da capacitação com os agentes comunitários de saúde (ACS) e profissionais da educação infantil.

$\mathrm{O}$ aumento da renda provoca aumento no consumo da maior parte dos alimentos ( $\mathrm{p}<$ 0.05 ) (Tabela 2), exceto para aqueles dos grupos dos açúcares $(\mathrm{p}=0.302)$, tubérculos $(\mathrm{p}=0.120)$, farinhas, féculas e massas $(\mathrm{p}=0.271)$ e sal e condimentos $(\mathrm{p}=0.181)$, que não foram diferentes entre os estratos, e para cereais e leguminosas, que mostraram declínio com o aumento da renda (de $38,6 \mathrm{Kg}$ para $28,3 \mathrm{Kg}$, $\mathrm{p}=0.003$ e de $13,5 \mathrm{Kg}$ para $9,4 \mathrm{Kg}, \mathrm{p}=0.001$, entre o segundo e terceiro estratos, respectivamente).

O consumo de frutas e legumes, embora aumente com a renda $(p=0.010$ e 0.003 entre os três estratos, dois a dois, respectivamente), é baixo (aproximadamente $140 \mathrm{~g}$ e $82 \mathrm{~g}$ per capita/dia, em média) e o de óleos e gorduras, bem como de sal e condimentos, elevado ( $32 \mathrm{~g}$ e $28 \mathrm{~g}$ per capital dia, respectivamente), considerando os valores médios entre os três estratos; entre o primeiro e o segundo estratos de renda há aumento do consumo de óleos e gorduras $(\mathrm{p}=0.016)$; já a quantidade de sal e condimentos não apresenta diferença entre os três estratos $(\mathrm{p}=0.181)$.

Quanto à contribuição energética, estima-se que cada indivíduo tenha cerca de $2000 \mathrm{Kcal}$ disponíveis no ambiente domiciliar por dia (Tabela $3)$. Os cereais representam a principal fonte energética da dieta, com 307,7Kcal (DP $=175,84)$, sem diferença entre os estratos de renda $(\mathrm{p}=$ 0.103). Em seguida, o principal grupo a fornecer energia são óleos e gorduras, com 272,3Kcal (DP $=131,66)$ e diferença estatística entre os dois primeiros estratos de renda $(\mathrm{p}=0.025)$. Açúcares, doces e produtos de confeitaria e panificados representam os dois grupos seguintes. 
Tabela 2. Consumo alimentar familiar per capita por ano, em kg. Centro de Saúde Perseu Leite de Barros. Campinas, 2009.

\begin{tabular}{|c|c|c|c|c|c|c|c|c|c|c|}
\hline \multirow{3}{*}{ Grupos de alimentos } & \multirow[b]{3}{*}{$\mathbf{n}$} & \multicolumn{9}{|c|}{ Aquisição alimentar domiciliar per capita anual (em kg) } \\
\hline & & \multicolumn{2}{|c|}{ Geral } & \multicolumn{2}{|c|}{ Bloco } & \multicolumn{2}{|c|}{$\begin{array}{c}\text { Bloco } \\
\text { SMFPC }=2\end{array}$} & \multicolumn{2}{|c|}{$\begin{array}{c}\text { Bloco } \\
\text { SMFPC }=3\end{array}$} & \multirow[b]{2}{*}{ valor $\mathbf{p}^{*}$} \\
\hline & & Média & DP & Média & $\mathrm{DP}$ & Média & DP & Média & DP & \\
\hline Laticínios & 181 & 70,70 & 36,98 & 57,81 & 42,07 & 70,45 & 35,87 & 78,07 & 35,96 & $0.036^{\mathrm{b}}$ \\
\hline Bebidas e infusões & 176 & 59,62 & 45,83 & 41,85 & 51,57 & 54,00 & 39,24 & 83,93 & 50,71 & $<0.001^{a, b, c}$ \\
\hline Frutas & 176 & 51,42 & 31,29 & 49,79 & 36,03 & 46,69 & 27,05 & 65,66 & 35,67 & $0.010^{b, c}$ \\
\hline Cereais & 175 & 34,96 & 18,27 & 30,28 & 15,36 & 38,60 & 19,48 & 28,28 & 13,85 & $0.003^{\mathrm{c}}$ \\
\hline Legumes & 181 & 29,84 & 19,02 & 25,77 & 19,21 & 28,05 & 17,79 & 37,09 & 20,69 & $0.003^{b, c}$ \\
\hline Carnes & 182 & 25,31 & 15,94 & 18,86 & 11,31 & 25,35 & 16,20 & 28,80 & 16,62 & $0.045^{\mathrm{b}}$ \\
\hline Açúcares, doces e produtos de confeitaria & 177 & 23,81 & 12,34 & 20,41 & 12,02 & 24,28 & 12,50 & 24,44 & 12,07 & 0.302 \\
\hline Panificados & 183 & 23,64 & 14,07 & 23,76 & 12,72 & 23,80 & 14,73 & 23,15 & 13,26 & 0.997 \\
\hline Tubérculos & 184 & 17,61 & 16,60 & 17,11 & 19,29 & 17,04 & 17,22 & 19,39 & 13,25 & 0.120 \\
\hline Hortaliças folhosas & 177 & 15,78 & 13,95 & 9,99 & 6,05 & 15,22 & 14,27 & 20,07 & 14,79 & $0.006^{\mathrm{b}, \mathrm{c}}$ \\
\hline Farinhas, féculas e massas & 179 & 13,54 & 8,00 & 12,02 & 8,71 & 14,10 & 8,36 & 12,93 & 6,53 & 0.271 \\
\hline Aves e ovos & 182 & 12,44 & 9,99 & 9,51 & 5,46 & 11,94 & 10,75 & 15,35 & 9,28 & $0.002^{b, c}$ \\
\hline Leguminosas & 179 & 12,20 & 7,37 & 10,95 & 3,72 & 13,48 & 8,20 & 9,43 & 5,41 & $0.001^{\mathrm{c}}$ \\
\hline Óleos e gorduras & 185 & 11,80 & 5,91 & 8,73 & 4,29 & 12,47 & 6,02 & 11,69 & 5,95 & $0.016^{\mathrm{a}}$ \\
\hline Sais e condimentos & 183 & 10,36 & 5,12 & 9,08 & 5,08 & 10,54 & 5,29 & 10,54 & 4,67 & 0.181 \\
\hline Alimentos preparados e misturas industriais & 5 & 4,88 & 7,34 & - & - & 1,62 & 0,97 & 9,78 & 11,56 & - \\
\hline Pescados & 126 & 4,24 & 5,36 & 1,95 & 1,81 & 3,75 & 4,12 & 6,34 & 7,73 & $0.001^{b, c}$ \\
\hline
\end{tabular}

"Resultado Análise de Variância para um fator (Anova) (significativo ao nível de 5\%, valor $\mathrm{p}<0,05)$. Teste de Tukey para múltiplas comparações:

a - diferença significativa entre Bloco 1 e Bloco 2; ${ }^{b}$ - diferença significativa entre Bloco 1 e Bloco 3; ${ }^{\text {c }}$ - diferença significativa entre Bloco 2 e Bloco 3.

Tabela 3. Consumo alimentar familiar: disponibilidade diária per capita de energia, em Kcal. Centro de Saúde Perseu Leite de Barros. Campinas, 2009.

\begin{tabular}{|c|c|c|c|c|c|c|c|c|c|c|}
\hline \multirow{3}{*}{ Grupos de alimentos } & \multirow[b]{3}{*}{$\mathbf{n}$} & \multicolumn{9}{|c|}{ Disponibilidade diária de energia per capita (Kcal) } \\
\hline & & \multicolumn{2}{|c|}{ Geral } & \multicolumn{2}{|c|}{ Bloco } & \multicolumn{2}{|c|}{$\begin{array}{c}\text { Bloco } \\
\text { SMFPC }=2\end{array}$} & \multicolumn{2}{|c|}{$\begin{array}{c}\text { Bloco } \\
\text { SMFPC }=3\end{array}$} & \multirow[b]{2}{*}{ valor $\mathrm{p}$} \\
\hline & & Média & DP & Média & DP & Média & DP & Média & DP & \\
\hline Cereais & 185 & 307,7 & 175,9 & 269,6 & 162,9 & 335,3 & 188,2 & 256,3 & 131,7 & 0.103 \\
\hline Óleos e gorduras & 185 & 272,3 & 131,1 & 204,3 & 102,8 & 286,4 & 129,3 & 272,4 & 140,0 & $0.025^{\mathrm{a}}$ \\
\hline Açúcares, doces e produtos de confeitaria & 185 & 234,5 & 127,5 & 197,4 & 115,4 & 241,5 & 131,9 & 236,6 & 121,4 & 0.223 \\
\hline Panificados & 185 & 208,8 & 117,9 & 208,2 & 114,7 & 208,9 & 122,2 & 208,9 & 110,2 & 0.949 \\
\hline Carnes & 184 & 181,6 & 116,9 & 144,3 & 105,8 & 184,3 & 121,7 & 195,1 & 107,6 & 0.090 \\
\hline Laticínios & 185 & 172,1 & 96,2 & 141,0 & 90,4 & 166,8 & 102,0 & 203,1 & 74,2 & $0.004^{b, c}$ \\
\hline Farinhas, féculas e massas & 181 & 137,9 & 84,7 & 120,7 & 87,7 & 144,6 & 90,4 & 129,9 & 65,2 & 0.239 \\
\hline Leguminosas & 182 & 110,2 & 68,7 & 101,6 & 34,5 & 121,7 & 76,7 & 83,6 & 49,6 & $<0.001^{\mathrm{c}}$ \\
\hline Bebidas e infusões & 184 & 99,1 & 91,4 & 87,4 & 96,1 & 95,6 & 87,0 & 115,2 & 100,1 & 0.247 \\
\hline Frutas & 184 & 86,0 & 61,7 & 76,5 & 58,6 & 78,0 & 53,9 & 111,9 & 75,1 & $0.002^{b, c}$ \\
\hline Aves e ovos & 185 & 60,2 & 62,5 & 44,6 & 25,1 & 60,2 & 72,6 & 68,8 & 43,9 & $0.014^{\mathrm{b}, \mathrm{c}}$ \\
\hline Tubérculos & 185 & 35,4 & 37,1 & 35,8 & 48,4 & 33,8 & 35,6 & 39,4 & 34,4 & 0.120 \\
\hline Sais e condimentos & 185 & 33,8 & 29,4 & 21,4 & 24,6 & 35,7 & 31,3 & 35,8 & 25,5 & $0.013^{\mathrm{a}, \mathrm{b}}$ \\
\hline Legumes & 184 & 23,5 & 16,5 & 19,4 & 14,6 & 21,4 & 13,7 & 31,4 & 21,5 & $0.001^{b, c}$ \\
\hline Alimentos preparados e misturas industriais & 5 & 21,0 & 12,4 & - & - & 16,0 & 9,2 & 28,5 & 16,2 & - \\
\hline Pescados & 126 & 14,6 & 24,0 & 7,2 & 5,3 & 12,1 & 10,9 & 23,4 & 41,6 & $0.004^{c}$ \\
\hline Hortaliças folhosas & 180 & 11,0 & 12,1 & 10,1 & 17,7 & 9,7 & 9,0 & 14,7 & 15,1 & $0.018^{c}$ \\
\hline
\end{tabular}

*Resultado Análise de Variância para um fator (Anova) (significativo ao nível de 5\%, valor p < 0,05). Teste de Tukey para múltiplas comparações:

a - diferença significativa entre Bloco 1 e Bloco 2; ${ }^{b}$ - diferença significativa entre Bloco 1 e Bloco 3; ${ }^{\mathrm{c}}$ - diferença significativa entre Bloco 2 e Bloco 3. 
O inventário de compras do mês possibilita comparação com estudos de âmbito nacional como a POF e permite identificar os grupos de alimentos que são priorizados no orçamento familiar. Alimentos de elevada densidade energética e baixa densidade nutricional, como amidos e óleos, respondem pelos quatro primeiros grupos em fornecimento de energia. Estudo semelhante conduzido em Campinas mostra que os mesmos alimentos, cereais, óleo e açúcar, são consumidos diariamente por famílias em insegurança alimentar muito grave ${ }^{21}$.

A vigilância dos dados de consumo realizada neste estudo, ao refletir a preferência das famílias em condição de vulnerabilidade, mostrou que pequenas alterações no repertório alimentar são possíveis por meio de oficinas sobre alimentação saudável e orientação na perspectiva de "melhores compras", que auxiliam as famílias a promover ajustes no consumo mensal, contribuindo para diminuição do consumo de sódio, açúcar, óleo e gorduras totais. Nas oficinas desenvolvidas em conjunto com as mães e agentes comunitários de saúde - ACS foram apresentadas receitas e modos de preparo rápidos com misturas constituídas por alimentos disponíveis na região e já integrantes de sua cesta de consumo. Os resultados do consumo alimentar familiar também foram discutidos durante as capacitações com ACS, profissionais da Equipe de SF, professores e monitores da creche situada na área de cobertura.

\section{O matriciamento da atenção nutricional - pressupostos teóricos}

Os dados epidemiológicos produzidos na fase de campo subsidiaram o entendimento sobre a situação das famílias no que se refere à alimentação e à nutrição nos três cenários de interesse para a formulação da proposta de matriciamento: comunidade, família e indivíduo?.

Segundo a Portaria do MS nº154 de $2008^{8}$, que cria os NASF, as ações de Alimentação e Nutrição devem ser voltadas para a promoção da alimentação saudável com base no resgate de práticas alimentares da região, acesso a alimentos locais de alto valor nutritivo e baixo custo, como também ações voltadas para as maiores demandas assistenciais, situadas na área materno-infantil e DCNT. Além disto, cada NASF deve estar vinculado a, no mínimo uma e no máximo nove Equipes de SF, de acordo com a portaria do MS n³124/2012 22. Esta modulação será tratada aqui, tendo em vista a experiência da fase de campo do estudo de matriciamento. Contudo, é patente que o aumento da demanda por ações de promoção da alimentação saudável como estratégia de prevenção e controle dos distúrbios nutricionais presentes na população, justifica a importância da $\mathrm{AB}$ e do foco nas ações de prevenção aos agravos à saúde, tanto devido às carências quanto aos excessos alimentares ${ }^{9,13,14,18,23}$. Ao considerar a proposição do Ministério da Saúde de que a $A B$ seja coordenadora das redes de atenção, que são organizações de conjuntos de serviços de saúde vinculados por uma missão única, por uma ação cooperativa e interdependente e por objetivos comuns para ofertar atenção contínua e integral a determinada população, fica demonstrada a importância do aprimoramento das ações da ESF e seus componentes, como os NASF, e da estruturação da $\mathrm{AB}$ para atender a este objetivo ${ }^{9}$.

$\mathrm{Na}$ presente investigação, experimentou-se o caminho de reconhecimento de território para subsidiar a aproximação com a ESF com vistas ao fortalecimento da prevenção e do monitoramento dos principais distúrbios nutricionais, de forma a qualificar a intervenção $0^{7,9,16}$. As disposições dos documentos oficiais sobre $\mathrm{AB}$ reafirmam que o nutricionista tem como prioridade atuar no âmbito familiar e comunitário e garantir a atenção nutricional individualizada de forma indireta, a partir da estruturação e organização de protocolos de atendimento e de encaminhamento ${ }^{9,16}$. A atualização da PNAN, ao ratificar a alimentação como direito da população e dever do Estado, indica que o setor saúde possua uma política específica em relação à alimentação e nutrição. A diretriz "Organização da atenção nutricional" reforça a importância de melhor organização dos serviços de saúde em atender às demandas geradas pelos agravos relacionados à alimentação e nutrição, por meio do apoio matricial e da clínica ampliada desenvolvidos pelas equipes multiprofissionais e oferecido às Equipes de $\mathrm{SF}^{12}$.

Outras referências sobre o rol das ações sob responsabilidade do nutricionista estão disponíveis em documentos como "O papel do nutricionista na Atenção Primária à Saúde" do Conselho Federal de Nutricionistas ${ }^{24}$, a "Matriz de ações de alimentação e nutrição na Atenção Básica"16 e "Diretrizes do NASF - Núcleos de Apoio à Saúde da Família"'; estas ações vão ao encontro do modelo proposto neste estudo, de forma a viabilizar as atividades para:

1. Realizar o diagnóstico nutricional e identificar a rede de apoio; durante o estudo, tanto escolas quanto outras estruturas disponíveis foram empregadas para o desenvolvimento das ações; 
2. Avaliar a segurança alimentar e nutricional - SAN, de forma a identificar precocemente riscos e dificuldades relacionados ao estado nutricional e à condição alimentar das famílias. Nesta investigação, que teve como foco famílias com crianças de 0 a 6 anos, incluiu-se a verificação do consumo mensal de alimentos; esta rotina mostrou ser de baixa complexidade e com bom potencial para a identificação de situações de insegurança alimentar;

3. Fomentar a educação alimentar a partir dos riscos identificados e privilegiar o consumo de alimentos regionais, bem como práticas de agricultura familiar; os resultados do estudo sobre consumo alimentar foram discutidos com as famílias; não se testou, contudo, ações dirigidas à agricultura familiar, dadas as características do território, situado na zona urbana do município e com poucas oportunidades de aproximação à produção de alimentos;

4. Participar dos programas de educação em saúde já instalados e desenvolvidos pelas Equipes de SF, de forma a contribuir com a promoção de práticas de alimentação saudável para todas as fases da vida e também instrumentalizar as equipes para esta formação; a equipe de pesquisa integrou-se à dinâmica da UBS por meio de participação em todas as reuniões com indicação de pauta de nutrição, além de ter promovido a construção das ações voltadas à identificação de risco nutricional lado a lado com os ACS;

5. Desenvolver os protocolos de atenção nutricional, quando aplicáveis, juntamente com a equipe de saúde, organizando o sistema de referência e contrarreferência; participar da elaboração dos planos terapêuticos nas reuniões de equipe; as ações para este propósito foram centrais no desenvolvimento do estudo, e mostraram uma excelente oportunidade para a construção de rotinas de atendimento apoiadas na complementaridade da atuação interdisciplinar, desde a identificação das situações de risco e reconhecimento do território até o estabelecimento de linhas de cuidado para interlocução com as ações de média e alta complexidade;

6. Capacitar as equipes de saúde sobre alimentação e nutrição, de forma continuada, especialmente os ACS que estão em contato com as famílias da área de cobertura das UBS; o estudo desenvolveu capacitação dos ACS sobre temas referentes à alimentação materno-infantil e promoveu a discussão dos resultados encontrados sobre estado nutricional da população infantil residente na área e do consumo alimentar familiar com toda equipe da UBS;
7. Desenvolver ações intersetoriais juntamente com as Equipes de SF e os NASF, envolvendo os setores de educação, assistência social, esporte, cultura e as redes de apoio da própria comunidade; o estudo promoveu atividades que contaram com a participação de profissionais da educação infantil e de pessoas da rede de apoio da comunidade.

No modelo proposto neste estudo, o nutricionista atuou como mediador do processo de educação alimentar da população da área de abrangência, a partir da compreensão da realidade discutida com o educando; ao conhecer os determinantes dos problemas nutricionais nas famílias e na comunidade, define as estratégias para aprimoramento das práticas alimentares daquela população em conjunto com a equipe de saúde ${ }^{24,25}$. A pesquisa exploratória inicial para o reconhecimento de território, que se estendeu por aproximadamente 18 meses, forneceu os elementos para o planejamento da etapa seguinte. Os indicadores nutricionais foram empregados para fortalecer conceitos de boas compras - com o propósito de favorecer escolhas em detrimento de alimentos como óleo, sal e fontes de açúcar - sobretudo nos estratos de menor poder aquisitivo; houve também prioridade para o tema alimentação complementar, tendo em vista a frequência de gestantes no pré-natal em ações que podem ser programadas com a equipe prevendo participação pontual do nutricionista, que não está inteiramente dedicado a cada ESF. A frequência às oficinas foi possível graças à intervenção dos Agentes Comunitários de Saúde e de parceria estabelecida com equipamentos sociais do bairro, como as paróquias e as escolas.

Assim, atividades de intervenção foram construídas a partir do contexto do educando na tentativa de valorização do seu saber, tornando-o protagonista de seu aprendizado e dando a ele a oportunidade de encontrar caminhos possíveis para a mudança, respeitando a não neutralidade do indivíduo e estimulando sua capacidade de sonhar e modificar hábitos de sua vida e das pessoas ao seu redor ${ }^{26,27}$.

A atuação a partir do patrimônio de cada família, valorizando seu universo e seus conhecimentos, promove mudanças de comportamento e maior aproximação no cuidado de seus membros; desta forma, envolve a comunidade para o exercício de seus direitos e para a garantia da SAN. Esta compreensão dialoga com a experiência de Margolis et al. ${ }^{28}$, bem sucedida em programa de promoção da saúde infantil a partir da integração da equipe com a comunidade e com 
experiências nacionais de protagonismo popular, consolidada em comunidades de São Paulo, Minas Gerais e Alagoas ${ }^{24}$.

\section{O modelo}

A implantação dos NASF demanda a revisão das formas de inserção profissional de cada membro das equipes a fim de oferecer serviços com diferentes graus de complexidade e fortalecer a atuação em rede, conceito que orienta a reorganização da atenção em saúde e ao mesmo tempo em que dialoga com o conceito de núcleo e campo proposto por Campos e Domitti ${ }^{7}$. Neste sentido, o matriciamento valoriza tanto ações de caráter preventivo quanto reparador, em que a contribuição da especialidade, usada aqui como expressão do acúmulo do saber em cada área, toma forma em uma perspectiva de atenção interdisciplinar em todos os níveis do Sistema ${ }^{8,9}$.

Apesar do foco do estudo ter sido o risco à saúde da criança de até 6 anos, foram identificadas as competências adequadas à atuação matricial do nutricionista na ESF, em alinhamento às propostas previstas na Matriz das ações de Alimentação e Nutrição na atenção básica ${ }^{16}$, que podem ser aplicadas a outras demandas por atenção nutricional, como aquelas decorrentes das DCNT.

O modelo matricial para a inserção de ações de nutrição e dietética para o combate aos principais distúrbios nutricionais na Estratégia Saúde da Família está apoiado na compreensão dos seguintes conceitos:

1. Atenção nutricional. Conjunto de ações para a promoção da saúde a partir de medidas profiláticas ou terapêuticas de cunho alimentar. Decorre de diagnóstico nutricional e da clínica ampliada. Em uma compreensão ampla do objeto de trabalho do nutricionista é a reunião das ações que integram a assistência dietética, e inclui ações de vigilância e linhas de cuidado.

2. Diagnóstico nutricional. Melhor conclusão sobre o estado nutricional consolidada a partir de indicadores antropométricos, socioeconômicos, de ingestão alimentar, clínicos e/ou bioquímicos, adotados de forma isolada ou complementar segundo escala de relevância e viabilidade.

3. Vigilância alimentar e nutricional. Acompanhamento da situação de alimentação e nutrição em determinada área de cobertura e de seus determinantes e condicionantes; integrada às demais ações de vigilância em saúde, ratifica a res- ponsabilidade integral da Equipe de SF com sua assistência, e subsidia o planejamento das ações por parte de gestores e da comunidade.

4. Linha de cuidado nutricional. Ação ou conjunto de ações organizadas com o objetivo de dar resposta a uma demanda alimentar e/ou nutricional. Inclui a identificação de sinais e sintomas, a elaboração de diagnóstico, a definição das estratégias dietéticas ou dietoterápicas e estabelece metas de caráter profilático ou terapêutico adequadas à situação socioeconômica e cultural do indivíduo, da família e da comunidade; prevê ações coordenadas nos diferentes níveis de atenção.

O modelo, ilustrado na Figura 1, tem por base o matriciamento das ações de nutrição experimentadas neste estudo, organizadas nos três cenários de atuação: o indivíduo, a família e a comunidade.

Para cada conjunto de metas, foram identificados: fontes, cenários e estratégias, organizados em três ciclos de atuação, de ocorrência simultânea e permanente.

A sistematização de ações organizadas em níveis de atenção valoriza a assistência nutricional, que pode contribuir para o enfrentamento do complexo perfil epidemiológico que se desenhou com o aumento da prevalência das DCNT, além de buscar formas de equacionar restrições de ordem orçamentária. O matriciamento das ações de assistência nutricional procura evidenciar formas para promover a saúde como consequência a uma política de promoção de bem-estar e qualidade de vida, entendidos como valores de equidade social, a exemplo do que propuseram Assis et al. ${ }^{23}$ em artigo que tratou da inserção do nutricionista na ESF.

\section{Os Ciclos do modelo de matriciamento para a atenção nutricional na Atenção Básica}

No Ciclo I, a meta é o reconhecimento do território. A consulta a dados secundários obtidos por meio de estudos de base populacional fornece elementos sobre condições de vida relevantes: escolaridade, renda e saneamento básico, entre outros. Na unidade e no Distrito de Saúde, o nutricionista pode recuperar informações sobre morbidade e a organização das Equipes de SF. Esta etapa constituiu a fase de pesquisa exploratória, com duração de 12 meses. A UBS em que a equipe do projeto atuou é composta por 3 Equipes de SF, com um total de 35 profissionais. A partir do diagnóstico da situação de saúde e de nutrição da população da área de cobertura, por 
CICLO I

Meta: reconhecimento do território

1. Serviço
Ârea de cobertura
População atendida
Número e organização das UBS
Número e organização das ESF
Número e organização dos NASF
Indicadores de saúde

. Indicadores IBGE ou PNAD

IDH

Renda

Escolaridade

Saneamento básico

Indicadores de saúde

\section{CICLO II}

Meta: Promoção de Segurança Alimentar e Nutricional e do Direito Humano à Alimentação Adequada; conferir autonomia e protagonismo para a prática alimentar saudável

1. Indivíduo

Diagnóstico Nutricional

Intervenção alimentar dietética

ou dietoterápica

Eventual referência para outros níveis do SUS

\section{Estratégias}

Linhas de cuidado

Projetos terapêuticos

Ambulatório com Clínica

Ampliada

Grupos educativos

\begin{tabular}{|l|}
\hline \multicolumn{1}{|c|}{} \\
2. Família \\
Estrutura para compra e \\
preparo de alimentos \\
Rede de apoio \\
Inventário de consumo mensal \\
Participação em programas \\
\hline
\end{tabular}

\section{Estratégias}

Visita domiciliar

Linhas de cuidado

Oficinas

Grupos educativos

\begin{tabular}{|l|}
\hline \multicolumn{1}{|c|}{} \\
\hline 3. Comunidade \\
Redes e estruturas de apoio \\
Lideranças \\
Equipamentos de varejo \\
\hline $\begin{array}{l}\text { Estratégias } \\
\text { Parcerias com escolas, creches, } \\
\text { varejistas } \\
\text { Linhas de cuidado } \\
\text { Oficinas }\end{array}$ \\
\hline
\end{tabular}

\begin{tabular}{|c|c|c|c|}
\hline \multicolumn{4}{|c|}{$\begin{array}{c}\text { CICLO III } \\
\text { Meta: intersetorialidade, corresponsabilização e resolubilidade }\end{array}$} \\
\hline$\nabla$ & $\nabla$ & $\nabla$ & $\nabla$ \\
\hline $\begin{array}{l}\text { 1. Fortalecer o } \\
\text { controle social } \\
\text { Ampliar o acesso a } \\
\text { políticas públicas }\end{array}$ & $\begin{array}{l}\text { 2. Vigilância } \\
\text { Responsabilização } \\
\text { pela área de cobertura }\end{array}$ & $\begin{array}{l}\text { 3. Sistema de } \\
\text { referência } \\
\text { Consolidar o } \\
\text { matriciamento com } \\
\text { o profissional de } \\
\text { referência }\end{array}$ & $\begin{array}{l}\text { 3. Sistema de } \\
\text { referência } \\
\text { Consolidar o } \\
\text { matriciamento com } \\
\text { o profissional de } \\
\text { referência }\end{array}$ \\
\hline $\begin{array}{l}\text { Estratégia } \\
\text { Participação e/ou } \\
\text { acompanhamento } \\
\text { do trabalho em } \\
\text { Conselhos }\end{array}$ & $\begin{array}{l}\text { Estratégia } \\
\text { Bancos de dados } \\
\text { SISVAN }\end{array}$ & $\begin{array}{l}\text { Estratégia } \\
\text { Clínica ampliada }\end{array}$ & $\begin{array}{l}\text { Estratégias } \\
\text { Ações educativas para } \\
\text { profissionais }\end{array}$ \\
\hline
\end{tabular}

Figura 1. Esquema ilustrativo do modelo para o matriciamento da atenção nutricional na Atenção Básica. Campinas, 2011.

meio do reconhecimento do território e a identificação das situações de risco e do estabelecimento de vínculo com as ESF e com a rede de apoio, foram desenvolvidas as atividades de campo.

O Ciclo II abriga as ações para promoção de Segurança alimentar e Nutricional (SAN) e Direito Humano à Alimentação Adequada (DHAA); organizadas nos três cenários, a atenção nutricional visa garantir autonomia e protagonismo para a prática alimentar saudável. O primeiro cenário contempla as ações de atenção nutricional dirigidas ao indivíduo, com foco no apoio à equipe de saúde para os casos que se reconhece demanda para a Nutrição, a partir dos protocolos de refe- 
rência e contrarreferência incluindo os níveis de média e alta complexidade do Sistema, quando aplicáveis. A relação com os níveis de média e alta complexidade para a assistência, contudo, não foi experimentada neste estudo, o que representa uma das fragilidades da investigação.

$\mathrm{O}$ atendimento ambulatorial clássico, embora possa compor a atenção nutricional, perde a centralidade na organização da rotina do nutricionista na $\mathrm{AB}$. A prescrição dietética individualizada tem lugar para acolher situações de maior complexidade que mereçam atenção, em uma perspectiva que promova clínica ampliada ${ }^{7,29}$, processo de trabalho que tem grande identificação com a atenção nutricional, uma vez que a abordagem via aproximação ao sujeito - e não à doença - está na sua essência. Assim, é a partir da escuta sensivel que a intervenção do nutricionista toma corpo e se estabelece a ação em que profissional e sujeito (paciente) definem a proposta terapêutica. É importante ratificar a competente anotação da conduta em prontuário: diagnóstico e intervenção alimentar, com registro das estratégias dietéticas adotadas.

O segundo cenário compreende as ações de atenção nutricional com a família, que se desenvolvem a partir da compreensão dos distúrbios nutricionais e dos fatores de risco associados. Neste cenário, o conceito "melhores compras" foi um dos focos de abordagem da atenção nutricional experimentado, além da avaliação do estado nutricional, que neste estudo considerou as crianças menores de 6 anos. A partir da análise do banco de dados, experimentou-se entregar aos responsáveis de cada criança um diagnóstico sobre a avaliação nutricional que informava sobre a necessidade, ou não, do acompanhamento em consulta com pediatra e a equipe local de saúde na UBS, para a abordagem do distúrbio nutricional instalado (baixo peso, baixa estatura e sobrepeso/obesidade).

A realização de oficinas de alimentação saudável foi a estratégia empregada pelos pesquisadores para divulgar os resultados relativos ao consumo familiar que contextualizam o ambiente nutricional da população em estudo. Além da demonstração dos riscos do excesso de consumo de sal, açúcar, doces, refrigerantes e embutidos - identificados no estudo de consumo familiar como de elevado consumo - a oficina tratou das recomendações para melhoria do padrão de consumo alimentar e de melhores compras. No estudo, foi formulada uma cesta básica saudável para atender a $70 \%$ das recomendações nutricionais de energia de uma família referência (composta por dois adultos, uma criança e um adolescente, alinhada ao perfil da localidade).

A identificação de estruturas de apoio, como igrejas, associação de bairro e escolas na área de abrangência, amplia a possibilidade de realizar oficinas culinárias. $\mathrm{Na}$ experiência do estudo, ao acompanhar e participar do preparo, cada integrante degustou as preparações; foram registrados os relatos quanto ao grau de complexidade da preparação, momento em que o nutricionista pôde disseminar boas práticas de manipulação de alimentos. A manipulação do alimento e o preparo da receita aproximam os participantes, ao empregar uma linguagem atraente, desconstruindo a verticalização das relações entre equipe de saúde e comunidade. Neste estudo, foram realizadas 5 oficinas, com a participação direta de 30 famílias. As famílias foram selecionadas para participar das oficinas, a partir do banco dos dados coletados na fase exploratória e de acordo com a demanda levantada pela equipe SF, especialmente pelos ACS.

Completa o Ciclo II o conjunto de atividades dirigidas à comunidade. Neste cenário, o objetivo é consolidar o serviço de saúde como referência para ações não apenas de caráter ambulatorial, mas também como promotor de qualidade de vida por meio de atividades coletivas, como as experimentadas nesta investigação. São exemplos de ações:

- Atenção ao pré-natal com inserção do nutricionista em dois eixos de ação. No primeiro, integrou-se à rotina do serviço de grupo educativo com a equipe de enfermagem e ACS, com temas sobre alimentação da gestante e estímulo ao AM, tratados por meio de discussão da realidade das participantes, depoimentos de experiências anteriores, em uma perspectiva de problematização. O segundo eixo de inserção se deu durante o pré-natal e a visita ao recém-nascido, oportunidade para ratificar as orientações e esclarecer as dúvidas em relação à alimentação na gestação e lactação. Os atendimentos ocorreram em dois momentos da gestação: primeiro e/ou segundo trimestres, e terceiro trimestre; os temas escolhidos com potencial para promoção do AM e da saúde da gestante foram: alimentação saudável na gestação e preparo de mamas para o AM, e Aleitamento materno: técnica e incentivo. Os dados das gestantes atendidas foram coletados da "Caderneta da Gestante" e inseridos em uma planilha para subsidiar a agenda de visita ao recém-nascido (RN). Após o nascimento da criança ocorreu uma visita domiciliar junto com o ACS à mãe e ao RN para avaliar a adesão às orientações. Neste 
Equipamentos de varejo e condições de abastecimento. O Ciclo II pode ainda incluir o monitoramento da disponibilidade de equipamentos de varejo na área de cobertura. Neste estudo, os pontos de varejo empregados para o abastecimento das famílias foram identificados em um mapa. Esta ação aproxima o nutricionista do repertório alimentar adotado pela família e permite o acompanhamento do custo da alimentação. Em conjunto com a Vigilância Sanitária, o nutricionista pode desenvolver ações indutoras de política de vendas de alimentos saudáveis e controle sanitário junto aos varejistas.

O Ciclo III do modelo aponta as ações de caráter intersetorial, no desenvolvimento da corresponsabilização pela população atendida entre UBS e NASF, em busca da resolubilidade das demandas do serviço de saúde. Entre estas ações destaca-se a Capacitação em Alimentação e Nutrição. Neste estudo, a deficiência da formação dos ACS em relação ao tema Alimentação e Nutrição levou a equipe a desenvolver uma capacitação nesta área, além da elaboração de um roteiro para identificação de situações de risco nutricional nas visitas domiciliares.

Por ser considerado o elo de ligação entre a família e o serviço de saúdes, o ACS é o profissional que pode auxiliar na identificação de situações de risco nutricional. A adequada formação deste profissional propicia a superação de limites impostos pela prática da equipe, ampliando sua atuação junto à população e favorecendo a adesão dos usuários às ações e serviços de saúde, como já se demonstrou em outros estudos ${ }^{30-32}$.

A capacitação foi desenvolvida em dois dias com duração de 3 horas/dia, a partir da exposição dos temas selecionados (Aleitamento materno e Alimentação complementar; Avaliação de risco nutricional; Desnutrição e Obesidade) e da problematização de situações relacionadas a estes mesmos temas pelos ACS e pela equipe de saúde. Esta abordagem tem como objetivo permitir que o educando seja o sujeito ativo de seu aprendizado, construindo significados e definindo representações da realidade de acordo com suas experiências; a partir da crítica e reflexão, o educando

passa a equacionar os problemas encontrados em sua realidade ${ }^{26}$.

Ao final da capacitação, foi realizado o treinamento com os ACS para a aplicação do roteiro de identificação de situações de risco nutricional nas visitas domiciliares, desenvolvido a partir das experiências de trabalho entre o nutricionista e o ACS com questões alimentares relacionadas ao domicílio e à família. Ao trabalhar com o PSF, Fernandez et al. ${ }^{18}$ identificaram deficiências nas ações de vigilância nutricional e também propuseram um modelo para treinamento dos ACS.

A realização do estudo em uma UBS que experimentava a presença do nutricionista pela primeira vez mostrou que a incorporação da atenção nutricional, vencidas as resistências "de chegada" pela presença do novo profissional, é fortemente dependente da relação com o ACS, antes de qualquer outro profissional da ESF. O resultado, contudo, depois de quase dois anos de trabalho conjunto, comprova a aceitação e o empoderamento que esta relação pode trazer para ambos os profissionais. O vídeo produzido neste estudo "O Agente de Saúde a atenção nutricional: uma perspectiva promissora" teve o objetivo de ilustrar por meio de linguagem leve, em que bases as competências do ACS podem apoiar o nutricionista para promoção de Segurança Alimentar e Nutricional.

Nesta direção, a atenção nutricional traz importante contribuição para a garantia do DHAA. Os três ciclos que integram o modelo proposto neste estudo organizam o trabalho do nutricionista de modo a compatibilizar a atual estrutura do SUS, especialmente no que se refere aos limites do seu financiamento, com a ampliação da equipe de profissionais. $\mathrm{O}$ entendimento de que promover o DHAA e a SAN está para além da concepção mais tecnicista da vigilância alimentar e nutricional, apoiada em quantificações de valor epidemiológico - cuja relevância não está em dúvida foi tratada por Bosi ${ }^{33}$ e por Prado et al. ${ }^{34}$, ao discutirem o lugar da pesquisa qualitativa e da produção do conhecimento em nutrição, sobretudo no campo da Saúde Coletiva. O matriciamento da assistência nutricional não nega a contribuição dos indicadores clássicos do diagnóstico nutricional (antropométricos, clínicos, de consumo e bioquímicos), mas relativiza seu espaço na rotina do nutricionista ligado à $\mathrm{AB}$, ao ratificar a contribuição da abordagem interdisciplinar e do trabalho do nutricionista com o ACS, a intersetorialidade e o fortalecimento do sistema de referência e contrarreferência com a atenção na média e na alta complexidade, abrindo espaço para o estudo do 
território e do fortalecimento da rede de atenção que inclui estes níveis do Sistema.

Neste sentido, a contribuição do modelo proposto procura trazer ao debate uma forma de proporcionar a inserção, na $\mathrm{AB}$, de um profissional que tem, na sua prática, a integração das dimensões biológica, social e ambiental, como tratado por Vasconcelos ${ }^{35}$ ao discutir o "corpo de conhecimentos científicos que garantiu especificidade ao campo da Nutrição no Brasil".

Contudo, os desafios que permeiam a atuação do nutricionista e demais profissionais do Núcleo de Apoio à Saúde da Família são a articulação interdisciplinar e a lógica de funcionamento do apoio matricial, de forma que a equipe SF compreenda o trabalho desta equipe NASF, que é apoio assistencial e técnico-pedagógico, centrado na promoção de saúde.

A reorientação da política nacional de ensino superior no que se refere à expansão e ao acompanhamento dos cursos de graduação e o ajustamento das Diretrizes Curriculares da área de Nutrição constituem estratégias que merecem fortalecimento para a compreensão ampliada da $\mathrm{AB}$ como cenário de atuação profissional ${ }^{36} \mathrm{e}$ contribuem para a garantia do direito legítimo da sociedade em contar com a contribuição dos saberes do nutricionista, como defendido por Assis et al..$^{23}$.

Neste sentido, o desafio para a implementação de um modelo como o aqui proposto está, especialmente, na revisão dos processos de trabalho dos profissionais de saúde na $A B$, em que ainda perdura o modelo biomédico. A ampliação das ações, sobretudo de caráter preventivo, emergentes de campos de atuação não médicos como a nutrição, demanda o desenho claro das formas de inserção compatíveis com a realidade do Sistema. A atenção nutricional constitui oportunidade para a proposta de um modelo ampliado de saúde e cuidado, no qual a integralidade da atenção considera componentes de ordem subjetiva, afetiva e social, além dos determinantes do processo de doença.

\section{Conclusão}

A atenção nutricional matricial pressupõe ações diferentes e adequadas para cada um dos focos: indivíduo, família e comunidade. Durante a realização do trabalho, foram eventuais as demandas por atendimento ambulatorial; em que pese sua importância, tem lugar periférico na rotina da nutrição na Atenção Básica; os processos de trabalho do nutricionista são majoritariamente destinados à Educação em Saúde, seja por meio de atividades em grupo (nas UBS ou em equipamentos comunitários), atividades em parceria com outras estruturas de apoio social (escolas, associações de moradores, igrejas, entre outras formas de organização), visitas domiciliares e educação continuada com ACS e outros profissionais da Equipe de SF. Estas ações fortalecem os mecanismos de participação e controle social e a intersetorialidade.

O sistema de referência e contrarreferência com os demais níveis de atenção do SUS para o equacionamento de questões de maior complexidade e o apoio dos ACS para identificação das situações de risco são duas pontas da atuação da ESF, reforçadas pela rede de atenção à saúde.

A inserção matricial de um nutricionista em jornada de 40 horas para dar apoio a cerca de 6 Equipes de SF, ou duas UBS de pequeno porte, ou ainda para uma área de cobertura de aproximadamente 20 mil habitantes foi experimentada na fase de campo deste estudo, desenvolvido em zona urbana de cidade de grande porte da região Sudeste. Não se tem conhecimento de outra experiência com o mesmo propósito. Por este motivo, este parece ser o produto mais relevante do trabalho: uma perspectiva que ilumina formas de desenvolver assistência nutricional a partir da experiência da clínica ampliada e do conhecimento do território, abordagens afeitas à natureza do trabalho do nutricionista. 


\section{Colaboradores}

M Borelli participou da coleta e análise dos dados, concepção e redação do artigo; SMA Domene participou da concepção, redação e revisão do artigo; LA Mais e J Pavan participaram da coleta e análise dos dados; JAAC Taddei participou da concepção e revisão do artigo. Todos os autores aprovaram a versão final do manuscrito.

\section{Referências}

1. Programa Saúde da Família. Informes Técnicos Institucionais. Departamento de Atenção Básica. Secretaria de Políticas de Saúde. Rev Saude Publica 2000; 34(3):316-319.

2. Brasil. Ministério da Saúde (MS). Secretaria de Atenção à Saúde. Departamento de Atenção Básica. Guia prático do Programa Saúde da Família. Brasília: MS; 2002.

3. Rosa WAG, L’Abate RC. Programa Saúde da Família: a construção de um novo modelo de assistência. Rev Latino- Am Enfermagem 2005; 13(6):1027-1034.

4. Oliveira EM, Spiri WC. Programa Saúde da Família: a experiência de equipe multiprofissional. Rev Saude Publica 2006; 40(4):727-733.

5. Brasil. Ministério da Saúde (MS). Secretaria de Atenção à Saúde. Departamento de Atenção Básica. Política Nacional de Atenção Básica. 4ª ed. Brasília: MS; 2007.

6. Campos GWS. Saúde pública e saúde coletiva: campo e núcleo de saberes e práticas. Cien Saude Colet 2000; 5(2):219-230.

7. Campos GWS, Domitti AC. Apoio matricial e equipe de referência: uma metodologia para gestão do trabalho interdisciplinar em saúde. Cad Saude Publica 2007; 23(2):399-407.

8. Brasil. Ministério da Saúde. Portaria no 154, de 24 de janeiro de 2008. Cria os Núcleos de Apoio à Saúde da Família - NASF. Diário Oficial da União 2008; 4 mar.

9. Brasil. Ministério da Saúde (MS). Secretaria de Atenção à Saúde. Departamento de Atenção Básica e Departamento de Ações Programáticas Estratégicas. Diretrizes do NASF - Núcleos de Apoio à Saúde da Família. Brasília: MS; 2010.

10. Brasil. Ministério da Saúde (MS). Departamento de Atenção Básica. Secretaria de Atenção à Saúde. Gráfico: Evolução do número de Núcleos de Apoio à Saúde da Família implantados, agosto de 2011 [acessado 2011 ago 28]. Disponível em: http://dab.saude.gov.br/abnumeros.php

11. Instituto Brasileiro de Geografia e Estatística (IBGE). Pesquisa de orçamentos familiares 2002-2003. Análise da disponibilidade domiciliar de alimentos e do estado nutricional no Brasil. Despesas, rendimentos e condição de vida. Rio de Janeiro: IBGE; 2004.

12. Brasil. Ministério da Saúde (MS). Secretaria de Atenção à Saúde. Departamento de Atenção Básica. Política nacional de alimentação e nutrição. Brasília: MS; 2011.

13. Recine E, Vasconcellos AB. Políticas nacionais e o campo da Alimentação e Nutrição em Saúde Coletiva: cenário atual. Cien Saude Colet 2011; 16(1):73-79.

14. Coutinho JG, Gentil PC, Toral N. A desnutrição e a obesidade no Brasil: o enfrentamento com base na agenda única da nutrição. Cad Saude Publica 2008; 24(Supl. 2):S332-S340.

15. Instituto Brasileiro de Geografia e Estatística (IBGE). Resultados do universo Censo 2000 [acessado 2008 ago 15]. Disponível em: http://www.ibge.gov.br/ibgecidades

16. Brasil. Ministério da Saúde (MS). Secretaria de Atenção à Saúde. Departamento de Atenção Básica. Matriz das ações de alimentação e nutrição na atenção básica de saúde. Brasília: MS; 2009. 
17. Brasil. Centro Brasileiro de Análise e Planejamento. Pesquisa Nacional de Demografia e Saúde da Mulher e da Criança - PNDS 2006. Brasília: Ministério da Saúde; 2008 [acessado 2010 jul 3]. Disponível em: www.saude. gov.br/pnds2006

18. Fernandez PMF, Voci SM, Kamata LH, Najas MS, Souza ALM. Programa Saúde da Família e as ações em nutrição em um distrito de saúde do município de São Paulo. Cien Saude Colet 2005; 10(3):749-755.

19. Brasil. Ministério da Saúde (MS). II Pesquisa de prevalência do aleitamento materno nas capitais e no Distrito Federal. Brasília: Disponível em UNICEF; 2009. (Série C. Projetos, Programas e Relatórios). [acessado 2010 jul 13]. Disponível em: http://bvsms.saude.gov. br/bvs/publicacoes/pesquisa_prevalencia_aleitamento _materno.pdf

20. Rocha S. Pobreza e indigência no Brasil - algumas evidências empíricas com base na PNAD 2004. Nova Economia Belo Horizonte 2006; 16(2):265-299.

21. Panigassi G, Segall-Correa AM, Marin-Leon L, Perez -Escamilla R, Maranha LK, Sampaio MFA. Insegurança alimentar intrafamiliar e perfil de consumo de alimentos. Rev Nutr 2008; 21(Supl.):135s-144s.

22. Brasil. Ministério da Saúde. Portaria nº n³124/2012, de 28 de dezembro de 2012. Redefine os parâmetros de vinculação dos Núcleos de Apoio à Saúde da Família (NASF) Modalidades 1 e 2 às Equipes Saúde da Família e/ou Equipes de Atenção Básica para populações específicas, cria a Modalidade NASF 3, e dá outras providências. Diário Oficial da União 2012; 29 dez.

23. Assis AMO, Santos SMC, Freitas MCS, Santos JM, Silva MCM. O Programa Saúde da Família: contribuições para uma reflexão sobre a inserção do nutricionista na equipe multidisciplinar. Rev Nutr 2002; 15(3):255-266.

24. Conselho Federal de Nutricionistas. O papel do nutricionista na Atenção Primária à Saúde [internet]. Documento de outubro de 2008 [acessado 2008 out 20]. Disponível em: www.cfn.org.br

25. Sawaya SM. Vencendo a desnutrição: abordagem pedagógica. São Paulo: Salus Paulista; 2002.

26. Westphal MF, Lima CWV, Duarte MF. Educação nutricional: uma abordagem de processo. In: Taddei JAAC organizador. Jornadas científicas do NISAN: Núcleo Interdepartamental de Segurança Alimentar e Nutricional 2004/2005. Barueri: Minha Editora; 2007. p. 152-163.

27. Freire P. Pedagogia da autonomia: saberes necessários à prática educativa. 40a ed. São Paulo: Paz e Terra; 1996.

28. Margolis PA, Stevens R, Bordley WC, Stuart J, Harlan C, Keyes-Elstein L, Wisseh S. From concept to application: the impact of a community-wide intervention to improve the delivery of preventive services to children. Pediatrics 2001; 108(3):e42.
29. Brasil. Ministério da Saúde. Secretaria de Atenção à Saúde. Núcleo Técnico da Política Nacional de Humanização (MS). Clínica ampliada, equipe de referência e projeto terapêutico singular. $2^{\text {a }}$ ed. $1^{\text {a }}$ reimp. Brasília: MS; 2008. ( Série B. Textos Básicos de Saúde)

30. Nunes MO, Trad LB, Almeida BA, Homem CR, Melo MCIC. O agente comunitário de saúde: construção da identidade desse personagem híbrido e polifônico. Cad Saude Publica 2002; 18(6):1639-1646.

31. Nascimento EPL, Correa CRS. O agente comunitário de saúde: formação, inserção e práticas. Cad Saude Publica 2008; 24(6):1304-1313.

32. Melo MB, Brant LC, Oliveira LA, Santos APS. Qualificação de agentes comunitários saúde: instrumento de inclusão social. Trab Educ Saúde (Online) 2010; 7(3):463-477.

33. Bosi MLM. Desafios na interface pesquisa-construção do direito humano à alimentação: reflexões no âmbito da segurança alimentar e nutricional. Cien Saude Colet 2010; 15(1):19-30.

34. Prado S, Gugelmim AS, Matos RA, Silva KJ, Olivares PSG. A pesquisa sobre segurança alimentar e nutricional no Brasil de 2000 a 2005: tendências e desafios. Cien Saude Colet 2010; 15(1):7-18.

35. Vasconcelos FAG. A ciência da nutrição em trânsito : da nutrição e dietética à nutrigenômica. Rev Nutr 2010; 23(6):935-945

36. Simonard-Loureiro HM, Schwarzschild LFCP, Tuma RCFB, Domene SMA. Nutrição. In: Haddad AE, Pierantoni CR, Ristoff D, Xavier I M, Giolo J, Silva LB, editores. A trajetória dos cursos de graduação na área da saúde: 1991-2004. Brasília: Instituto Nacional de Estudos e Pesquisas Educacionais Anísio Teixeira; 2006. p. 353-379 [acessado 2011 ago 10]. Disponível em: http:// bvsms.saude.gov.br/bvs/publicacoes/Texto_de_Referencia.pdf

Artigo apresentado em 16/09/2014

Aprovado em 13/12/2014

Versão final apresentada em 15/12/2014 